\title{
Supersymmetry enhancement and junctions in S-folds
}

\author{
Yosuke Imamura, ${ }^{a}$ Hirotaka Kato ${ }^{a}$ and Daisuke Yokoyama ${ }^{b}$ \\ ${ }^{a}$ Department of Physics, Tokyo Institute of Technology, \\ 2-12-1 Ookayama, Meguro-ku, Tokyo, Japan \\ ${ }^{b}$ Department of Mathematics, King's College London, \\ The Strand, London, WC2R 2LS U.K. \\ E-mail: imamura@phys.titech.ac.jp, h.kato@th.phys.titech.ac.jp, \\ daisuke. yokoyama@kcl.ac.uk
}

Abstract: We study supersymmetry enhancement from $\mathcal{N}=3$ to $\mathcal{N}=4$ proposed by Aharony and Tachikawa by using string junctions in S-folds. The central charges carried by junctions play a central role in our analysis. We consider planer junctions in a specific plane. Before the S-folding they carry two complex central charges, which we denote by $Z$ and $\bar{Z}$. The S-fold projection eliminates $\bar{Z}$ as well as one of the four supercharges, and when the supersymmetry is enhanced $\bar{Z}$ should be reproduced by some non-perturbative mechanism. For the models of $\mathbb{Z}_{3}$ and $\mathbb{Z}_{4}$ S-folds which are expected to give $\mathrm{SU}(3)$ and $\mathrm{SO}(5) \mathcal{N}=4$ theories we compare the junction spectra with those in perturbative brane realization of the same theories. We establish one-to-one correspondence so that $Z$ coincides. By using the correspondence we also give an expression for the enhanced central charge $\bar{Z}$.

KEYWords: D-branes, Extended Supersymmetry

ARXIV EPRINT: 1606.07186 


\section{Contents}

1 Introduction 1

2 Junctions and BPS conditions 3

2.1 Junctions in S-folds 3

2.2 BPS bounds 5

3 Matching of charges and moduli $r$

$\begin{array}{lll}3.1 & \mathbb{Z}_{3} \text { S-fold } & 9\end{array}$

$\begin{array}{lll}3.2 & \mathbb{Z}_{4} \text { S-fold } & 12\end{array}$

$\begin{array}{lll}3.3 & \mathbb{Z}_{6} \text { S-fold } & 17\end{array}$

4 Walls of marginal stability 18

$\begin{array}{llr}5 & \text { Discussions } & 20\end{array}$

\section{Introduction}

A superconformal algebra in four dimensions is specified by the number of supercharges, $\mathcal{N} \leq 4$. Theories with $\mathcal{N}=1,2,4$ have been studied extensively in the last few decades, and played essential roles in the development of field theories. $\mathcal{N}=3$ theories, however, had not attracted much attention until quite recently. A reason for this is that the only $\mathcal{N}=3$ multiplet of free fields is the vector multiplet, which has the same contents as the $\mathcal{N}=4$ vector multiplet, and if we try to make an $\mathcal{N}=3$ theory in a perturbative way we always end up with an $\mathcal{N}=4$ theory. This means if there exist genuine $\mathcal{N}=3$ theories they should necessarily be strongly coupled. This is consistent to the fact that the absence of marginal deformations in $\mathcal{N}=3$ theories $[1,2]$.

An explicit construction of $\mathcal{N}=3$ theories was recently proposed by García-Etxebarria and Regalado in [3]. ${ }^{1}$ They realize $\mathcal{N}=3$ theories on a stack of D3-branes by performing a $\mathbb{Z}_{k}$ orbifolding that project out one of four supercharges on the D3-branes. Such an orbifold can be defined by combining $\mathrm{U}(1)_{R}$ symmetry of type IIB supergravity $[5,6]$ and a rotation of the transverse space $\mathbb{R}^{6}$. The $\mathrm{U}(1)_{R}$ is broken by the quantization of $(p, q)$ string charges down to a discrete subgroup depending on the value of the complex field $\tau=\chi+i e^{-\phi}$. For generic $\tau$ the subgroup is $\mathbb{Z}_{2}$ and then the orbifolded theory is still $\mathcal{N}=4$, while for some special values $\mathbb{Z}_{k}$ with $k=3,4,6$ are unbroken and then we can define genuine $\mathcal{N}=3$ theories. The resulting orbifolds, which we call S-folds following [7], are labelled by two integers $k$ and $\ell ; k$ is the order of the orbifold group and $\ell$ is a divisor

\footnotetext{
${ }^{1}$ See also [4] for an attempt of studying $\mathcal{N}=3$ theories by using AdS/CFT correspondence.
} 


\begin{tabular}{|cc|}
\hline$(k, \ell, N)$ & $G$ \\
\hline$(k, 1,1)$ & $\mathrm{U}(1)$ \\
\hline$(3,1,2)$ & $\mathrm{SU}(3)$ \\
$(4,1,2)$ & $\mathrm{SO}(5)$ \\
$(6,1,2)$ & $G_{2}$ \\
\hline
\end{tabular}

Table 1. Theories in which $\mathcal{N}=3$ is enhanced to $\mathcal{N}=4$ are shown.

of $k$ related to the discrete torsion. Therefore, the theory on the D3-branes are labeled by $k, \ell$, and $N$, where $N$ is the number of mobile D3-branes.

Aharony and Tachikawa [7] listed the dimensions of the Coulomb branch operators in these theories. ${ }^{2}$ In particular, they contain an operator with dimension $N \ell$, which becomes 1 or 2 for some of the theories. Aharony and Evtikhiev showed in [1] that the dimensions of Coulomb branch operators in a genuine $\mathcal{N}=3$ theory must be equal to or greater than 3. This was confirmed for rank- $\mathcal{N}=3$ theories in [8, 9]. This fact implies that the $\mathcal{N}=3$ theories with $N \ell=1$ or 2 are in fact $\mathcal{N}=4$ theories [7], which can be specified by giving the gauge group $G$. In [7] some BPS states were identified with 1/2 BPS W-bosons, and the gauge groups were determined (table 1 ).

The purpose of this paper is to analyze the spectra of these theories. Because each theory is defined by a system of D3-branes in an S-fold, we can realize dyonic particles by string junctions [10-14] connecting D3-branes and the S-plane.

This analysis is highly non-trivial. Let us consider $(k, \ell, N)=(k, 1,1)$ case. As is pointed out in [7], this should give the $\mathcal{N}=4$ Maxwell theory. We can consider an open $(p, q)$ string connecting a D3-brane and one of its mirror branes. Although it seems to give massive BPS states with non-vanishing dyonic charge in a generic point of the Coulomb branch there are no such massive BPS states in the $\mathcal{N}=4$ Maxwell theory. This implies that the string should be decoupled in the field theory limit. One may think that the central charge of a string is proportional to its length, and we should have light massive BPS states when the D3-brane is sufficiently close to the S-plane. However, the central charge gives only a lower bound of the mass, and does not guarantee the existence of the light states. We consider planar junctions in a specific plane. Before the $\mathbb{Z}_{k}$ S-folding we can define two complex central charges $Z$ and $\bar{Z}$ carried by junctions. One of them, say $\bar{Z}$, is projected out in the S-fold. In the system with the supersymmetry enhancement, the central charge $\bar{Z}$ revives non-perturbatively, and it may give a bound greater than the perturbative one. In this case the naive expectation about the presence of light BPS states would fail.

\footnotetext{
${ }^{2}$ In $[7]$ it is pointed out that the theories on the S-folds are obtained by gauging certain discrete symmetries of parent theories. A parent theory includes operators charged under the discrete symmetry, and in the corresponding gauged theory they are projected out and do not appear in the spectrum of gauge invariant operators. The current of enhanced supersymmetry is such an operator, and the theories we study in this paper are not the gauged theories but parent ones. Our analysis in the following section is not affected by this difference.
} 
Unfortunately, in the present paper, we cannot directly determine the non-perturbative bound. Instead, we determine it by using another realization of the theory in which the $\mathcal{N}=4$ symmetry is manifest. Some of the gauge groups in table 1 are classical Lie groups, and such $\mathcal{N}=4$ theories have a simpler brane realization, in which $\mathcal{N}=4$ supersymmetry and the associated central charges are realized perturbatively. We first compare the central charges $Z$ that is realized perturbatively both in the S-fold and in the perturbative set-up. With this information we establish relations between Coulomb moduli and dyonic charges in the S-fold and those in the other set up. Then, by assuming the equivalence of the two theories, we guess an expression of the non-perturbative central charge $\bar{Z}$ in terms of the Coulomb moduli and the dyonic charges of the particle.

This paper is organized as follows.

In the next section we study general aspects of S-folds and junctions in them. Constraints imposed on the charges of strings ending on S-planes and the perturbative BPS bound in S-folds are given. In section 3 we give one-to-one correspondence between the charge lattice of junctions in the S-fold realization and that in a perturbative realization of $\mathcal{N}=4 \mathrm{SU}(3)$ and $\mathrm{SO}(5)$ theories. The structure of a lattice is related non-trivially to the properties of the S-plane or O-plane which exists in the set-up, and the successful matching seems to strongly support the conjecture of the supersymmetry enhancement. Furthermore, we propose a formula to determine central charges without referring to the simpler realization. It gives $Z$ and $\bar{Z}$ even in the $G_{2}$ case, which does not have perturbative string realization.

In section 4 we carry out a preliminary analysis of walls of marginal stability [14]. We focus on strings in the $\mathbb{Z}_{3}$ and $\mathbb{Z}_{4}$ S-folds that connect a D3-brane and one of its mirror, and determine walls in Coulomb branch moduli space based on the assumption of the supersymmetry enhancement. The results are different from what are expected by perturbative analysis on the S-folds.

Section 5 is devoted to discussions.

\section{Junctions and BPS conditions}

In this subsection we will consider junctions in S-folds.

Let us first consider junctions in a system of $n$ parallel D3-branes in the flat background without any S-folding. A junction $\boldsymbol{j}$ is specified by charges $\left(p_{a}, q_{a}\right)(a=1, \ldots, n)$ of strings ending on the D3-branes. For strings ending on mobile D3-branes we always define string charges as incomming charges. When we discuss S-folds later it is more convenient to use the complex charges $Q_{a}=p_{a}+\tau q_{a}$. The charge conservation requires $Q_{a}$ to satisfy

$$
\sum_{a=1}^{n} Q_{a}=0 .
$$

When the positions of D3-branes are generic the low-energy effective theory of the parallel D3-branes is the $\mathcal{N}=4 \mathrm{U}(1)^{n}$ supersymmetric gauge theory. Let $\mathrm{U}(1)_{a}$ be the gauge 
group corresponding to the $a$-th D3-brane. In the low-energy effective theory a junction is regarded as a particle with the $\mathrm{U}(1)_{a}$ dyonic charge $Q_{a}$.

Let us consider junctions in $\mathbb{Z}_{k}$ S-fold. For $k \geq 3$ we set $\tau=\gamma \equiv e^{2 \pi i / k}$, while for $k=2$ we can take an arbitrary $\tau$. Let $\Gamma_{k}$ be the lattice on the complex plane spanned by 1 and $\tau$.

We introduce $N$ D3-branes on the S-fold. As in the case of orientifolds it may be possible to introduce D3-branes that are mirror to themselves. We introduce them later as a non-trivial discrete torsion. Here we assume the absence of such trapped D3-branes at the fixed point. Then in the covering space we have $k N$ D3-branes. We label them by two indices $a=1, \ldots, N$ and $i=0, \ldots, k-1$, where $a$ labels $N$ independent branes and $i$ labels $k$ branes identified by $\mathbb{Z}_{k}$ action. Their positions $z_{i, a}$ are related by

$$
z_{i, a}=\gamma^{i} z_{a}
$$

where $z_{a} \equiv z_{0, a}$. As in the flat background a junction is specified by the string charges $Q_{i, a} \in \Gamma_{k}$ on the D3-branes. We define $\mathbb{Z}_{k}$ action so that it rotates the coordinate $z$ and the complex charge $Q$ in the opposite direction by the same angle as given in (2.17). Due to the $\mathbb{Z}_{k}$ identification a string with charge $Q_{i, a}$ attached on the brane at $z_{i, a}$ is equivalent to a string with charge $\gamma^{i} Q_{i, a}$ attached on the brane at $z_{a}$. When we read off the $\mathrm{U}(1)^{N}$ dyonic charges $Q_{a}$ we should take account of this equivalence. We collect the endpoints on $z_{i, a}$ with $i=0, \ldots, k-1$ to the brane with $i=0$ by $\mathbb{Z}_{k}$ transformations and obtain

$$
Q_{a}=\sum_{i=0}^{k-1} \gamma^{i} Q_{i, a}
$$

These charges are again elements of $\Gamma_{k}$. We have assumed trivial discrete torsion, and there are no D3-branes at the origin of the covering space. In this case a junction is attached on only mobile D3-branes and due to the charge conservation $Q_{i, a}$ must satisfy

$$
\sum_{i, a} Q_{i, a}=0
$$

Due to this constraint $Q_{a}$ defined by (2.3) may not be all independent. The constraint is obtained as follows.

Let $F_{k}$ be a $\mathbb{Z}_{k}$ invariant homomorphism from $\Gamma_{k}$ onto some discrete group $K$. The $\mathbb{Z}_{k}$ invariance means $F_{k}(\gamma Q)=F_{k}(Q)$ for an arbitrary $Q \in \Gamma_{k}{ }^{3}$ By using $(2.3),(2.4)$, and the $\mathbb{Z}_{k}$ invariance of $F_{k}$ we can easily show

$$
F_{k}\left(Q_{0}\right)=0
$$

\footnotetext{
${ }^{3}$ The group $K$ is easily obtained as follows. Let us consider $k=2$ case as an example. Then $\Gamma_{2}$ is the lattice generated by 1 and $\tau$. Let $a$ and $b$ be two elements of $K$ corresponding to them: $a=F_{2}(1)$ and $b=F_{2}(\tau)$. By the linearity $F_{2}(p+q \tau)=p a+q b(p, q \in \mathbb{Z})$, and the $\mathbb{Z}_{2}$ invariance requires $2 a=2 b=0$. This means $K$ contains at most four elements $0, a, b$, and $a+b$. If these are all different we obtain the maximal $K\left(=\mathbb{Z}_{2}+\mathbb{Z}_{2}\right)$. If one of $a, b$, and $a+b$ is zero $K=\mathbb{Z}_{2}$, and if $a=b=0$ then $K=0$.
} 


\begin{tabular}{|ccccc|}
\hline$k$ & 2 & 3 & 4 & 6 \\
\hline$\Gamma^{\text {tor }}$ & $\mathbb{Z}_{2}+\mathbb{Z}_{2}$ & $\mathbb{Z}_{3}$ & $\mathbb{Z}_{2}$ & 0 \\
\hline
\end{tabular}

Table 2. The discrete torsion groups for $\mathbb{Z}_{k}$ S-folds are shown.

where $Q_{0}$ is the total charge

$$
Q_{0}=\sum_{a=1}^{N} Q_{a}
$$

If $K$ is not trivial (2.5) gives a non-trivial constraint imposed on the set of the charges $Q_{a}$. On the S-fold, $Q_{0}$ can be regarded as the charge of a string attached on the S-plane. (For S-planes and O-planes we define the charge of a string ending on them as the outgoing charge.) Therefore, (2.5) gives the constraint on the string charge that can be attached on the S-plane.

The constraint (2.5) is a necessary condition. There may be some different choices of $K$, and for some of them (2.5) may not be a sufficient one. However, we can easily show that if we choose maximal $K(2.5)$ gives the sufficient condition for the existence of junctions. The maximal $K$ are in fact isomorphic to the discrete torsion group

$$
\Gamma^{\text {tor }}=H^{3}\left(\boldsymbol{S}^{5} / \mathbb{Z}_{k}, \widetilde{\mathbb{Z}+\mathbb{Z}}\right)
$$

for the $\mathbb{Z}_{k}$ S-fold $[7,15,16]$ (table 2).

Up to here we consider S-folds with the trivial discrete torsion. Introduction of a nonvanishing discrete torsion changes the condition given above. To determine the modified condition it is convenient to realize such an S-plane by using wrapped fivebranes. (Similar realization of O-planes with non-trivial discrete torsions was given in $[15,17]$.) By the Poincare duality the discrete torsion can be also regarded as the two-cycle homology. Let us consider an S-fold with a torsion $t \in \Gamma^{\text {tor }}$. We can realize such an S-fold from that with the trivial torsion by wrapping a fivebrane around the two-cycle specified by $t$. (Note that the coefficient group $\widetilde{\mathbb{Z}+\mathbb{Z}}$ is the sheaf of the $(p, q)$ charges of fivebranes and an element of $\Gamma^{\text {tor }}$ specifies not only the cycle wrapped by the fivebrane but also the fivebrane charges.) The existence of the wrapped $(p, q)$ fivebrane allows $(p, q)$ strings to end on it. Namely, string with charge $Q_{0}$ can be attached on the S-plane if

$$
F\left(Q_{0}\right) \in \mathbb{Z} t
$$

The right hand side is not just $t$ but $\mathbb{Z} t$ because we can attach an arbitrary number of strings.

\subsection{BPS bounds}

Let us consider supersymmetry and BPS bounds in $\mathcal{N}=4$ theories. We denote the four supercharges with the positive chirality by $Q_{\alpha}^{I}$ and their Hermitian conjugate by $\bar{Q}_{\dot{\alpha} I}$. The indices $\alpha=1,2$ and $\dot{\alpha}=\dot{1}, \dot{2}$ are left-handed and right-handed spinor indices for $\mathrm{SO}(1,3)_{0123}$, respectively. The upper and lower indices $I=1,2,3,4$ label the 
fundamental and the anti-fundamental representations of $\mathrm{SU}(4)_{R} \sim \mathrm{SO}(6)_{456789}$. Their anti-commutation relations are

$$
\left\{Q_{\alpha}^{I}, \bar{Q}_{\dot{\beta} J}\right\}=\sigma_{\alpha \dot{\beta}}^{\mu} \delta_{J}^{I} P_{\mu}, \quad\left\{Q_{\alpha}^{I}, Q_{\beta}^{J}\right\}=\epsilon_{\alpha \beta} \rho_{m}^{I J} Z^{m+3},
$$

where $Z^{m+3}(m=1, \ldots, 6)$ are central charges belonging to the vector representation of $\mathrm{SU}(4)_{R}$, and $\rho_{m}^{I J}$ is an $\mathrm{SU}(4)_{R}$ invariant tensor with two anti-symmetric fundamental indices and one vector index. The central charges $Z^{m+3}$ form a complex vector in the $\mathbb{R}^{6}$ transverse to the D3-branes, and its real and imaginary part can be interpreted as the extension of fundamental strings and that of D-strings, respectively, in the brane realization of the $\mathcal{N}=4$ SYM.

The BPS bound obtained from (2.9) is [18]

$$
m^{2} \geq|\operatorname{Re} \vec{Z}|^{2}+|\operatorname{Im} \vec{Z}|^{2}+|\operatorname{Re} \vec{Z}||\operatorname{Im} \vec{Z}| \sin \alpha,
$$

where $\alpha$ is the angle between two vectors $\operatorname{Re} \vec{Z}$ and $\operatorname{Im} \vec{Z}$ in $\mathbb{R}^{6}$. For a junction to be BPS and to saturate this bound it must be planar $[18,19]$. We consider planer junctions in 89 plane, and it is convenient to decompose the $\mathrm{SO}(6)_{456789}$ fundamental representation into $\mathrm{SO}(4)_{4567} \times \mathrm{SO}(2)_{89}$ representations as

$$
\mathbf{4}=(\mathbf{2}, \mathbf{1})_{+\frac{1}{2}}+(\mathbf{1}, \mathbf{2})_{-\frac{1}{2}} \cdot
$$

We denote the supercharges belonging to $(\mathbf{2}, \mathbf{1})_{+1 / 2}$ and $(\mathbf{1}, \mathbf{2})_{-1 / 2}$ by $Q_{\alpha}^{a}$ and $Q_{\alpha}^{\dot{a}}$, respectively, and their Hermitian conjugate by $\bar{Q}_{\dot{\alpha} a}$ and $\bar{Q}_{\dot{\alpha} \dot{a}}$, respectively. If only two components $Z^{8}$ and $Z^{9}$ out of the six are non-vanishing, $Q_{\alpha}^{a}$ and $Q_{\alpha}^{\dot{a}}$ anticommute to each other. Then the algebra (2.9) splits into two copies of $\mathcal{N}=2$ algebra.

$$
\begin{aligned}
& \left\{Q_{\alpha}^{a}, \bar{Q}_{\dot{\beta} b}\right\}=\sigma_{\alpha \dot{\beta}}^{\mu} \delta_{b}^{a} P_{\mu}, \quad\left\{Q_{\alpha}^{a}, Q_{\beta}^{b}\right\}=\epsilon_{\alpha \beta} \epsilon^{a b} Z, \\
& \left\{Q_{\alpha}^{\dot{a}}, \bar{Q}_{\dot{\beta} \dot{b}}\right\}=\sigma_{\alpha \dot{\beta}}^{\mu} \delta_{\dot{b}}^{\dot{a}} P_{\mu}, \quad\left\{Q_{\alpha}^{\dot{a}}, Q_{\beta}^{\dot{b}}\right\}=\epsilon_{\alpha \beta} \epsilon^{\dot{a} \dot{b}} \bar{Z}
\end{aligned}
$$

where $Z=Z^{8}+i Z^{9}$ and $\bar{Z}=Z^{8}-i Z^{9}$. Note that $Z^{8}$ and $Z^{9}$ are complex, and $Z$ and $\bar{Z}$ are not conjugate to each other. $Z$ and $\bar{Z}$ carry $\mathrm{U}(1)_{R} \times \mathrm{SO}(2)_{89}$ charges $(+1,+1)$ and $(+1,-1)$, respectively. For an open $(p, q)$ string with the complex charge $Q=p+\tau q$ and the extension $\Delta z$ on the 89 plane these central charges are given by

$$
Z=Q \Delta z, \quad \bar{Z}=Q \Delta z^{*} .
$$

(Note that $Q$ and $\Delta z$ carry $\mathrm{SO}(2)_{R} \times \mathrm{SO}(2)_{89}$ charges $(+1,0)$ and $(0,+1)$, respectively.) Each $\mathcal{N}=2$ algebra in (2.12) gives the bound independently:

$$
m \geq|Z|, \quad m \geq|\bar{Z}| .
$$

For a string junction consisting of more than one open string the central charges of the junction are given by

$$
Z=\sum_{i} Q_{i} z_{i}, \quad \bar{Z}=\sum_{i} Q_{i} z_{i}^{*}
$$


where $z_{i}$ are the positions of D3-branes and $Q_{i}$ are complex charges of strings ending on them. If a junction contains more than two strings with different charges only one of the bounds in (2.14) can be saturated. To saturate the bound $m \geq|Z|$, all the constituent open strings should satisfy $m=|Z|$. This means that $\arg Z$ for the strings must be the same. Namely, the angles $\arg Q+\arg \Delta z$ are the same for all strings. We call such a junction "a holomorphic junction". On the other hand, for a junction saturating the bound $m \geq|\bar{Z}|$, the angles $\arg Q-\arg \Delta z$ are the same for all the the constituent strings. We call such a junction "an anti-holomorphic junction". ${ }^{4}$ Two bounds can be saturated at the same time only for a set of parallel strings with the same charge. Every state belongs to one of the following four types.

- $m=|Z|=|\bar{Z}|: 1 / 2$ BPS states

- $m=|Z|>|\bar{Z}|$ : holomorphic $1 / 4$ BPS states

- $m=|\bar{Z}|>|Z|$ : anti-holomorphic 1/4 BPS states

- $m>|Z|$ and $m>|\bar{Z}|$ : non-BPS states

Next, let us consider how these BPS bounds in $\mathcal{N}=4$ theory are modified when we perform an S-fold projection. An S-fold is defined by the projection which leaves states invariant under the $\mathbb{Z}_{k}$ action generated by

$$
g=\gamma^{J_{45}-J_{67}+J_{89}-R}
$$

where $J_{i j}$ and $R$ are generators of $\mathrm{SU}(4)_{R}$ and $\mathrm{U}(1)_{R}$, respectively. $g$ acts on the complex coordinate of the 89-plane $z$ and the complex string charge $Q$ as

$$
z \stackrel{g}{\longrightarrow} z^{\prime}=\gamma z, \quad Q \stackrel{g}{\longrightarrow} Q^{\prime}=\gamma^{-1} Q .
$$

The supercharges $Q^{a}$ and $Q^{\dot{a}}$ and the central charges $Z$ and $\bar{Z}$ are transformed as

$$
\left(Q^{1}, Q^{2}, Q^{\dot{1}}, Q^{\dot{2}}\right) \rightarrow\left(Q^{1}, Q^{2}, Q^{\dot{1}}, \gamma^{-2} Q^{\dot{2}}\right), \quad Z \rightarrow Z, \quad \bar{Z} \rightarrow \gamma^{-2} \bar{Z} .
$$

If $k \geq 3 Q^{\dot{2}}$ and $\bar{Z}$ are projected out and we obtain $\mathcal{N}=3$ theory. This theory is generically genuine $\mathcal{N}=3$ theory. However, there are cases that an additional supersymmetry is generated non-perturbatively, and what we are interested in are such cases. Because the algebra of this emergent $\mathcal{N}=4$ symmetry is isomorphic to the usual one, we have the same BPS bounds $m \geq|Z|$ and $m \geq|\bar{Z}|$. However, $\bar{Z}$ is not given by (2.15).

Before ending this section, we give a comment on the existence of BPS saturating states. For the $\mathcal{N}=4 \mathrm{SU}(N)$ SYM the BPS spectrum of dyons and the corresponding junctions have been studied in detail. A particle corresponding to a $(p, q)$ string is $1 / 2$ BPS and its ground states form a short multiplet. A particle realized as a 3-pronged junction has 12 fermionic zero-modes $[19,21,22]$ and its ground states form a multiplet of middle size. More general junctions have also been studied and, for example, a prescription to

\footnotetext{
${ }^{4}$ These two types of junctions are called class A and class B in [20].
} 
calculate their contribution to some BPS indices is known [20], and generically we have BPS saturating states.

However, in more general situation with S-plane or O-plane, this is not the case. Even if we can draw a junction whose constituent strings satisfy the condition we mentioned above, it does not mean the existence of BPS quantum states. For example let us consider a fundamental string connecting a D3-brane and its mirror in an orientifold background. Although it seems 1/2 BPS, it is indeed the case only when the RR-charge of the O-plane is positive. If the RR-charge is negative the BPS saturating states are projected out by the orientifold projection, and the mass of the string is on the order of string scale even if the D-branes coincide with the O-plane.

\section{$3 \quad$ Matching of charges and moduli}

Let us consider junctions in the $\mathbb{Z}_{k}$ S-fold with trivial discrete torsion. If we put two D3branes the supersymmetry is expected to be enhanced from $\mathcal{N}=3$ to $\mathcal{N}=4$ [7]. In this section we determine the both central charges $Z$ and $\bar{Z}$ for junctions in the S-folds by using relations to perturbative realization of the same $\mathcal{N}=4$ theories.

Following [7] let us begin with the identification of W-bosons in the $\mathbb{Z}_{k}$ S-fold. We consider an open string connecting two D3-branes. We denote a string with complex charge $Q$ that goes from a D3-brane at $z_{j, 2}$ to another D3-brane at $z_{i, 1}$ by

$$
z_{i, 1} \stackrel{Q}{\longleftarrow} z_{j, 2}, \quad Q \in \Gamma_{k} .
$$

Let $Q_{i, a}^{(S)}$ be the charges of strings attached on D3-branes in the covering space. (We put the superscript " $(S)$ " for distinction from the quantities in the dual set-up we will introduce later.) This is the junction with $Q_{i, 1}^{(S)}=Q, Q_{j, 2}^{(S)}=-Q$, and the other $Q_{i, a}^{(S)}$ vanishing. (2.3) gives

$$
\left(Q_{1}^{(S)}, Q_{2}^{(S)}\right)=\left(\gamma^{i} Q,-\gamma^{j} Q\right) .
$$

Following [7] we impose the following electric condition:

$$
Q_{1}^{(S)}=-Q_{2}^{(S) *}
$$

Then the string charge $Q$ must satisfy

$$
Q= \pm|Q| \gamma^{-\frac{i+j}{2}}
$$

and (3.2) becomes

$$
\left(Q_{1}^{(S)}, Q_{2}^{(S)}\right)= \pm|Q|\left(\gamma^{\frac{i-j}{2}},-\gamma^{\frac{j-i}{2}}\right) .
$$

Different $i-j$ gives different charges, and it is shown in [7] that these charges form the weight vectors in the adjoint representation of a rank-2 Lie group depending on $k$ (table 1 ).

In the following subsections, we give detailed analysis of the three models with supersymmetry enhancement. 


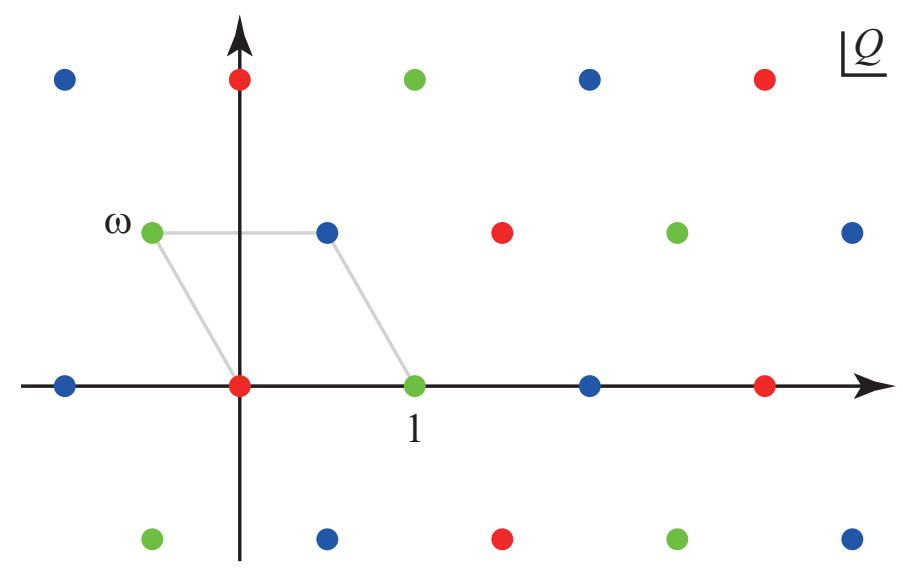

Figure 1. The $\mathbb{Z}_{3}$ grading of the lattice $\Gamma_{3}$ is shown. Red, green, and blue dots represent charges with $F(Q)=0,1$ and 2 , respectively.

\begin{tabular}{|ccccc|}
\hline & $\left(Q_{1}^{(S)}, Q_{2}^{(S)}\right)$ & $Z^{(S)}$ & $\left(Q_{1}^{(F)}, Q_{2}^{(F)}\right)$ & $Z^{(F)}$ \\
\hline $\boldsymbol{a}$ & $(1,-1)$ & $z_{1}-z_{2}$ & $(1,-1)$ & $y_{1}-y_{2}$ \\
$\boldsymbol{b}$ & $\left(\omega,-\omega^{2}\right)$ & $\omega z_{1}-\omega^{2} z_{2}$ & $(-1,0)$ & $-y_{1}$ \\
$\boldsymbol{c}$ & $\left(\omega^{2},-\omega\right)$ & $\omega^{2} z_{1}-\omega z_{2}$ & $(0,1)$ & $y_{2}$ \\
\hline
\end{tabular}

Table 3. The dyonic charges and the central charge of junctions in the S-fold and flat background are shown for three strings corresponding to W-bosons.

\section{$3.1 \quad \mathbb{Z}_{3}$ S-fold}

Let us first consider $\mathbb{Z}_{3}$ S-fold. In this case the modulous on the S-fold is $\tau^{(S)}=\omega=e^{2 \pi i / 3}$, and the complex string charges form the triangular lattice $\Gamma_{3}$ in figure 1 . (For $k=3$ we use $\omega$ instead of $\gamma$.) The charges $Q_{1}^{(S)}$ and $Q_{2}^{(S)}$ are elements of $\Gamma_{3}$, and constrained by (2.5),

$$
F_{3}\left(Q_{1}^{(S)}+Q_{2}^{(S)}\right)=0
$$

where $F_{3}$ is the map from $\Gamma_{3}$ onto $\mathbb{Z}_{3}$. This defines the $\mathbb{Z}_{3}$ grading in the lattice shown in figure 1. A string can end on the S-plane only when its charge is a red dot in figure 1.

Different choices of $j$ and $i$ in (3.5) with the same $j-i$ give equivalent strings mapped to one another by $\mathbb{Z}_{k}$, and only $j-i$ is significant. Let $\boldsymbol{a}, \boldsymbol{b}$, and $\boldsymbol{c}$ denote the strings corresponding to $j-i=0,1$, and 2 . In the $\mathbb{Z}_{3}$-frame in which the string charge becomes $Q=1$, these strings are

$$
\boldsymbol{a}: z_{1} \stackrel{1}{\longleftarrow} z_{2}, \quad \boldsymbol{b}: \omega z_{1} \stackrel{1}{\longleftarrow} \omega^{2} z_{2}, \quad \boldsymbol{c}: \omega^{2} z_{1} \stackrel{1}{\longleftarrow} \omega z_{2} .
$$

The dyonic charges and central charges of these strings are shown in table 3 . As is confirmed in [7] these are the same as those of W-bosons of SU(3) SYM.

To see this more clearly, let us use another realization of the $\mathcal{N}=4 \mathrm{SU}(3)$ theory with parallel D3-branes in the flat background without any S-folding. We use $y$ for the coordinate of D3-branes. The center of mass motion is decoupled and we take the coordinate so that 
one of the D3-branes is located at $y=0$, and denote the positions of the other two branes by $y_{1}$ and $y_{2}$. We denote the charges of the strings ending at $y_{1}$ and $y_{2}$ by $Q_{1}^{(F)}$ and $Q_{2}^{(F)}$, respectively. (The superscripts " $(F)$ " indicate the flat background.) By using these charges we can determine the central charge $Z^{(F)}$ by the general formula (2.15).

Let us assume that the strings in (3.7) correspond to fundamental strings stretched between two D3-branes on the $y$-plane. The sum of the charges of the three strings in (3.7) vanishes, and we choose the corresponding strings so that they also carry charges with vanishing sum:

$$
\boldsymbol{a}: y_{1} \stackrel{1}{\longleftarrow} y_{2}, \quad \boldsymbol{b}: 0 \stackrel{1}{\longleftarrow} y_{1}, \quad \boldsymbol{c}: y_{2} \stackrel{1}{\longleftarrow} 0
$$

The central charge $Z^{(F)}$ and the dyonic charges $\left(Q_{1}^{(F)}, Q_{2}^{(F)}\right)$ for these are shown in table 3 . Because all strings in (3.8) are fundamental ones, the central charge does not depend on the modulous $\tau^{(F)}$ on the flat background, which is not fixed yet.

We require $Z^{(S)}=Z^{(F)}$ for the W-boson states in table 3. This means

$$
Z=Q_{1}^{(F)} y_{1}+Q_{2}^{(F)} y_{2}=Q_{1}^{(S)} z_{1}+Q_{2}^{(S)} z_{2} .
$$

By requiring this equality for the $\mathrm{W}$-bosons we obtain the relations among $z_{a}$ and $y_{a}$ :

$$
z_{1}=\frac{\omega y_{1}+\omega^{2} y_{2}}{\omega-\omega^{2}}, \quad z_{2}=\frac{\omega^{2} y_{1}+\omega y_{2}}{\omega-\omega^{2}} .
$$

$Z^{(S)}$ and $Z^{(F)}$ are holomorphic functions in the complex charges. This means that $Q_{a}^{(S)}$ and $Q_{a}^{(F)}$ should be related by holomorphic linear relations. The comparison of the complex charges of $\mathrm{W}$-boson states in table 3 determines the following relations.

$$
Q_{1}^{(F)}=\frac{\omega Q_{1}^{(S)}+\omega^{2} Q_{2}^{(S)}}{\omega-\omega^{2}}, \quad Q_{2}^{(F)}=\frac{\omega^{2} Q_{1}^{(S)}+\omega Q_{2}^{(S)}}{\omega-\omega^{2}} .
$$

By taking account of $Q_{a}^{(S)} \in \Gamma_{3}$ and the constraint (3.6), we can show that $Q_{a}^{(F)}$ are also element of $\Gamma_{3}$ and no constraint is imposed. Furthermore, (3.11) is one-to-one. From the quantization of $Q_{a}^{(F)}$ we can fix the modulous on the flat background as $\tau^{(F)}=\omega$.

The other central charge $\bar{Z}$ can be directly obtained by the general formula (2.15) only in the flat background, and in the S-fold we cannot calculate it directly because it is generated non-perturbatively. However, once we obtain $\bar{Z}$ in the flat background, we can use the relations (3.10) and (3.11) to rewrite it as a function of the variables on the S-fold side. The result is

$$
\bar{Z}=Q_{1}^{(F)} y_{1}^{*}+Q_{2}^{(F)} y_{2}^{*}=-Q_{1}^{(S)} z_{2}^{*}-Q_{2}^{(S)} z_{1}^{*}
$$

At the second equality we used (3.10) and (3.11). On the S-fold side this is non-local in the sense that each term contains the charge and the coordinate of different branes.

For the following discussions the equation

$$
|Z|^{2}-|\bar{Z}|^{2}=\left(\left|Q_{1}^{(S)}\right|^{2}-\left|Q_{2}^{(S)}\right|^{2}\right)\left(\left|z_{1}\right|^{2}-\left|z_{2}\right|^{2}\right)
$$


may be convenient. By using this we can easily check that for an electric junction $\boldsymbol{w}$ with $Q_{2}^{(S)}=-Q_{1}^{(S) *}$ the absolute values of two central charges coincides; $|\bar{Z}[\boldsymbol{w}]|=|Z[\boldsymbol{w}]|$.

As a consistency check let us compare singularities in the moduli spaces of the two set-ups.

When $z_{1}=z_{2}, z_{1}=\omega z_{2}$, or $z_{1}=\omega^{2} z_{2}$, two D3-branes in the S-fold collide and the gauge symmetry is enhanced to $\mathrm{U}(2)$. These three singular loci correspond to $y_{1}=y_{2}$, $y_{1}=0$, and $y_{2}=0$, respectively, in the flat background. On these loci two of three D3-branes coincide, and the gauge symmetry is enhanced to $\mathrm{U}(2)$, just like on the S-fold side. This agreement of the loci is rather trivial because we determine the relations among moduli parameters by requiring the coincidence of the central charge $Z$ of string connecting two independent D3-branes, which vanishes if two D3-branes collide.

On the S-fold side, we have another type of singularity. When one of $z_{1}$ and $z_{2}$ approaches the S-plane, a string connecting the D3-brane and one of its mirrors shrinks to zero length, and appearance of massless particles is naively expected. This limit, however, causes no singularity on the flat background. When $z_{1}=0$ or $z_{2}=0$, three D3-branes in the flat background form an equilateral triangle, and no junction shrinks to zero size. In figure $2(\mathrm{a})$ we show two strings

$$
z_{1, a} \stackrel{1}{\longleftarrow} z_{2, a}, \quad(a=1,2),
$$

in a situation with $\left|z_{1}\right| \ll\left|z_{2}\right|$. The dyonic charges of these strings are

$$
\left(Q_{1}^{(S)}, Q_{2}^{(S)}\right)=\left\{\begin{array}{ll}
\left(\omega-\omega^{2}, 0\right) & (a=1) \\
\left(0, \omega-\omega^{2}\right) & (a=2)
\end{array} .\right.
$$

By the relation (3.11) we obtain the charges of the corresponding junctions:

$$
\left(Q_{1}^{(F)}, Q_{2}^{(F)}\right)=\left\{\begin{array}{ll}
\left(\omega, \omega^{2}\right) & (a=1) \\
\left(\omega^{2}, \omega\right) & (a=2)
\end{array} .\right.
$$

Junctions carrying these charges in the flat background are shown in figure 2(b). Although they have similar shapes the corresponding strings have completely different length in the S-fold. In particular, in the $z_{1}=0$ limit the junctions in 2(b) seem to have the same mass while in 2(a) the red one is massive and the blue one is massless.

One may think that all these strings/junctions are BPS, and naive estimation of masses should give the correct values. If it were the case, the above observation would mean inequivalence of theories given by two set-ups. This is, however, too naive.

Remember that for planar junctions on the 89 plane in the $\mathcal{N}=4$ theory there are two central charges $Z$ and $\bar{Z}$, which gives independent BPS bounds. The two junctions in figure $2(\mathrm{~b})$ are $1 / 4$ BPS configurations saturating different bounds; the red one is a holomorphic junction saturating $m=|Z|>|\bar{Z}|$ while the blue one is an anti-holomorphic junction saturating $m=|\bar{Z}|>|Z|$. On the S-fold, however, only $Z$ is realized perturbatively, and $\bar{Z}$ is generated by some unknown non-perturbative dynamics. From the length of strings in figure 2 (a) we can determine only $Z$ of two strings. Although they give the 


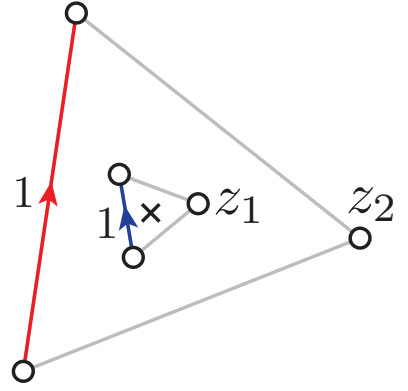

(a)

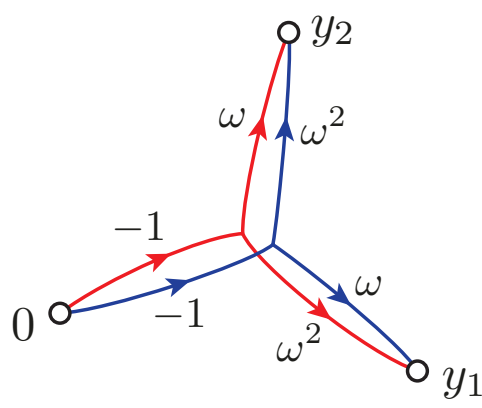

(b)

Figure 2. Examples of strings in the S-fold and corresponding junctions in the flat background.

lower bounds for the masses, the saturation is not guaranteed. If $|\bar{Z}|$ is greater than $|Z|$ the bound $m \geq|Z|$ is never saturated, and this is the case for the blue string in figure 2(a). We emphasize that there is no reason that the perturbative estimation gives good approximation of the mass. We consider the situation in which $z_{1}$ is much smaller than the string scale and the string coupling is of order 1 , and we cannot exclude large quantum corrections.

\section{$3.2 \quad \mathbb{Z}_{4}$ S-fold}

Next let us consider $\mathbb{Z}_{4}$ case. The modulus is $\tau=i$ and the complex charge of strings in the S-fold takes value in the square lattice $\Gamma_{4}=\{p+i q \mid p, q \in \mathbb{Z}\}$. If we put two D3-branes on this background it is expected to give the $\mathcal{N}=4 \mathrm{SO}(5)$ theory, which can also be realized by an orientifold. In this subsection we determine the spectrum of junctions in the S-fold and compare it with the spectrum on the orientifold side.

Before starting the analysis of the junction spectra let us briefly review some properties of O3-planes relevant to the analysis below. There are four types of O3-planes distinguished by the discrete torsion $t \in \mathbb{Z}_{2}+\mathbb{Z}_{2}$ of R-R and NS-NS three-form fluxes [15]. If we put D3-branes in an orientifold $\mathcal{N}=4$ theory with an orthogonal or symplectic gauge group is realized. The relation between the torsion and the gauge group is shown in table 4 . Among four types of O3-planes, the three but one with $t=(0,0)$ are transformed among them by $\mathrm{SL}(2, \mathbb{Z})$ symmetry of the type IIB string theory. Correspondingly, the gauge theories realized by them are equivalent via Montonen-Olive duality. In the case with two D3-branes, which we are interested in, the gauge groups $\mathrm{SO}(5)$ and $\mathrm{Sp}(2)$ are isomorphic, and the equivalence of these theories is trivial. However, the $\mathrm{W}$-bosons are realized in different ways. We refer to these two realization of the theory as $\mathrm{SO}(5)$ and $\mathrm{Sp}(2)$ theories.

Let us first determine the junction spectrum in the $\mathbb{Z}_{4}$ S-fold with two D3-branes. The charges of strings $Q_{a}^{(S)}(a=1,2)$ ending on the D3-branes satisfy

$$
F_{4}\left(Q_{1}^{(S)}+Q_{2}^{(S)}\right)=0
$$

where $F_{4}$ is the homomorphism from $\Gamma_{4}$ onto $\mathbb{Z}_{2}$ which define the $\mathbb{Z}_{2}$ grading of $\Gamma_{4}$ shown in figure 3. (3.17) shows that the charges $Q_{1}^{(S)}$ and $Q_{2}^{(S)}$ must have the same $\mathbb{Z}_{2}$ grading. 


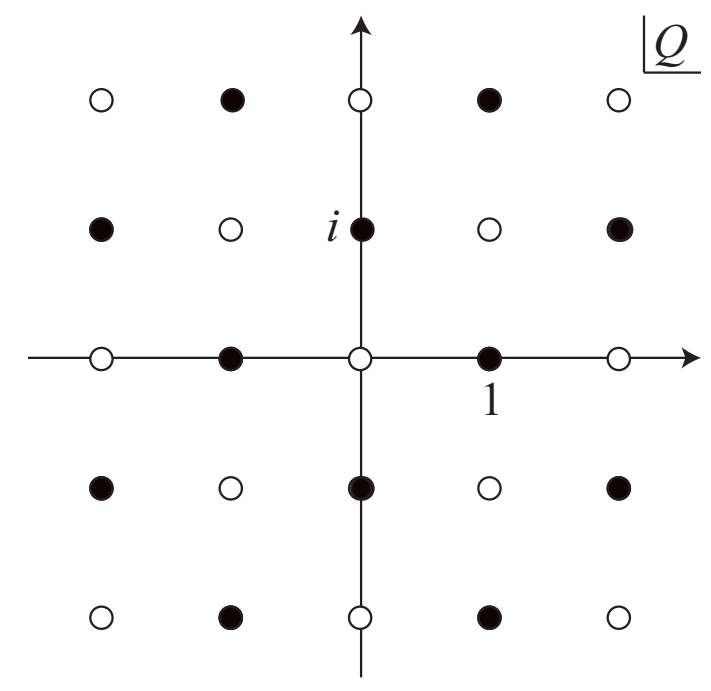

Figure 3. A $\mathbb{Z}_{2}$ grading of the lattice $\Gamma_{4}$ is shown. White and black dots represent charges with $F(Q)=0$ and 1 , respectively.

\begin{tabular}{|ccccc|}
\hline & $\mathrm{O}^{-}$ & $\widetilde{\mathrm{O}}^{-}$ & $\mathrm{O}^{+}$ & $\widetilde{\mathrm{O}}^{+}$ \\
\hline$t$ & $(0,0)$ & $(1,0)$ & $(0,1)$ & $(1,1)$ \\
$G$ & $\mathrm{SO}(2 N)$ & $\mathrm{SO}(2 N+1)$ & $\mathrm{Sp}(N)$ & $\mathrm{Sp}(N)$ \\
\hline
\end{tabular}

Table 4. The discrete torsions and the gauge groups realized by the orientifolds with four kinds of O3-planes are shown. The first and the second component of $t$ represent the torsions for R-R and NS-NS fluxes, respectively. $N$ is the number of the mobile D3-branes.

The strings whose charges satisfy the electric condition (3.3) are

$$
\boldsymbol{a}: z_{1} \stackrel{1}{\longleftarrow} z_{2}, \quad \boldsymbol{b}: z_{1} \stackrel{1-i}{\longleftarrow} i z_{2}, \quad \boldsymbol{c}: z_{1} \stackrel{i}{\longleftarrow}-z_{2}, \quad \boldsymbol{d}: z_{1} \stackrel{1+i}{\longleftarrow}-i z_{2}
$$

and ones with opposite orientation. We would like to identify these strings with W-bosons of $\mathrm{SO}(5)$ and $\mathrm{Sp}(2)$ theories that are realized on the orientifolds.

Let us consider a system with an O3-plane at $y=0$ and two D3-branes at $y=y_{1}, y_{2}$ (and their mirrors at $\left.y=-y_{1},-y_{2}\right)$. The complex charge of $(p, q)$-string is $Q^{(O)}=p+q \tau^{(O)}$. (The superscripts " $(O)$ " indicate orientifold.) We should notice that the modulus $\tau^{(O)}$ may not be the same as $\tau^{(S)}=i$. This should be determined so that the junction spectrum agrees with that of the S-fold. We denote complex charges of strings ending on the D3branes by $Q_{a}^{(O)}(a=1,2)$. Following $(2.6)$ we also define

$$
Q_{0}^{(O)}=Q_{1}^{(O)}+Q_{2}^{(O)}
$$

This is the charge of strings ending on the O3-plane. The general formula (2.8) says that a $(p, q)$ string can end on an O3-plane with $t \neq 0$ only when the charges satisfy

$$
(p, q)=0 \quad \text { or } \quad t \bmod 2 .
$$




\begin{tabular}{|ccccc|}
\hline & $Z^{(S)}$ & $\left(Q_{1}^{(S)}, Q_{2}^{(S)}\right)$ & $Z^{(O)}$ & $\left(Q_{1}^{(O)}, Q_{2}^{(O)}\right)$ \\
\hline $\boldsymbol{a}$ & $z_{1}-z_{2}$ & $(1,-1)$ & $-y_{1}$ & $(-1,0)$ \\
$\boldsymbol{b}$ & $(1-i)\left(z_{1}-i z_{2}\right)$ & $(1-i,-1-i)$ & $-y_{1}-y_{2}$ & $(-1,-1)$ \\
$\boldsymbol{c}$ & $i\left(z_{1}+z_{2}\right)$ & $(i, i)$ & $y_{2}$ & $(0,1)$ \\
$\boldsymbol{d}$ & $(1+i)\left(z_{1}+i z_{2}\right)$ & $(1+i,-1+i)$ & $-\left(y_{1}-y_{2}\right)$ & $(-1,1)$ \\
\hline
\end{tabular}

Table 5. The dyonic charges and the central charge of junctions in the S-fold and $\widetilde{\mathrm{O}}^{-}$-plane background are shown for four strings corresponding to W-bosons.

In particular, a fundamental string with charge $(1,0)$ can end on $\widetilde{\mathrm{O}}^{-}-$-plane, but not on $\mathrm{O} 3^{+}$- and $\widetilde{\mathrm{O} 3}{ }^{+}$-planes.

The W-bosons of $\mathrm{SO}(5)$ and $\mathrm{Sp}(2)$ realizations are given in a consistent way to this constraint. For an $\widetilde{\mathrm{O}}^{-}$-plane $\mathrm{SO}(5) \mathrm{W}$-bosons are given by open strings

$$
y_{2} \stackrel{1}{\longleftarrow} y_{1}, \quad-y_{1} \stackrel{1}{\longleftarrow} y_{2}, \quad 0 \stackrel{1}{\longleftarrow} y_{1}, \quad y_{2} \stackrel{1}{\longleftarrow} 0
$$

while for $\mathrm{O}^{+}{ }_{-}$and $\widetilde{\mathrm{O}}^{+}{ }^{+}$-planes $\mathrm{Sp}(2)$ W-bosons arise as strings

$$
y_{2} \stackrel{1}{\longleftarrow} y_{1}, \quad-y_{1} \stackrel{1}{\longleftarrow} y_{2}, \quad-y_{1} \stackrel{1}{\longleftarrow} y_{1}, \quad y_{2} \stackrel{1}{\longleftarrow}-y_{2} .
$$

We want to determine relations between strings (3.18) in the S-fold and (3.21) or (3.22) in the orientifold. The first two in (3.21) are the same with the first two in (3.22):

$$
y_{2} \stackrel{1}{\longleftarrow} y_{1}, \quad-y_{1} \stackrel{1}{\longleftarrow} y_{2} .
$$

Let us start with matching these two with two of the strings in (3.18). Because two strings in (3.23) are continuously deformed to each other by moving D3-branes, so are the corresponding strings. Among four strings in (3.18) $\boldsymbol{a}$ and $\boldsymbol{c}$ are deformed to each other, and so are $\boldsymbol{b}$ and $\boldsymbol{d}$, too. Therefore, we have only two essentially different choices.

First let us try matching strings in (3.23) with $\boldsymbol{d}$ and $\boldsymbol{b}$. Matching of these two is sufficient to determine the relation between the moduli parameters and that between dyonic charges. By identifying the central charges $Z^{(S)}$ and $Z^{(O)}$ we obtain the relations

$$
z_{1}=-\frac{1}{2}\left(y_{1}+i y_{2}\right), \quad z_{2}=\frac{1}{2}\left(y_{1}-i y_{2}\right) .
$$

From the comparison of charges we obtain

$$
Q_{1}^{(O)}=-\frac{1}{2}\left(Q_{1}^{(S)}-Q_{2}^{(S)}\right), \quad Q_{2}^{(O)}=-\frac{i}{2}\left(Q_{1}^{(S)}+Q_{2}^{(S)}\right) .
$$

These relations automatically fix the correspondents of $\boldsymbol{a}$ and $\boldsymbol{c}$ in (3.18), and we find that they correspond to the two remaining strings in (3.21). (See table 5.) Namely, this gives the correspondence to the adjoint representation in the $\mathrm{SO}(5)$ theory. 


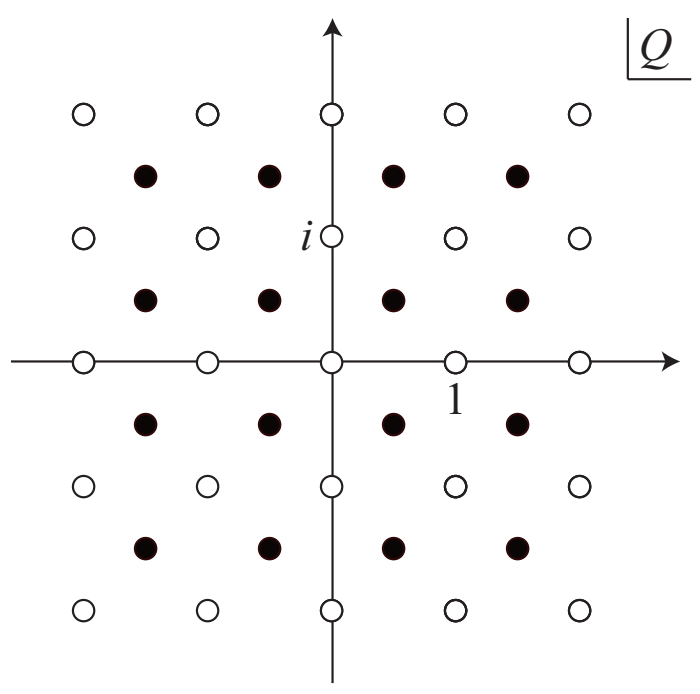

Figure 4. A $\mathbb{Z}_{2}$ grading of the lattice with $\tau=(1+i) / 2$ is shown. White and black dots represent charges with 0 and 1 in $\mathbb{Z}_{2}$, respectively.

$Q_{a}^{(S)}$ satisfying (3.17) can be given by

$$
Q_{a}^{(S)}=p_{a}+i q_{a}, \quad p_{1}+p_{2}+q_{1}+q_{2} \in 2 \mathbb{Z} .
$$

Substituting this into (3.25) we obtain

$$
Q_{1}^{(O)}=\frac{1}{2}\left[\left(p_{1}-p_{2}\right)+i\left(q_{1}-q_{2}\right)\right], \quad Q_{2}^{(O)}=\frac{1}{2}\left[\left(-q_{1}-q_{2}\right)+i\left(p_{1}+p_{2}\right)\right] .
$$

(3.26) implies that these charges take values in the $\mathbb{Z}_{2}$ graded lattice in figure 4 , and $Q_{1}^{(O)}$ and $Q_{2}^{(O)}$ have the same grading. The latter statement means that $Q_{0}^{(O)}$, the charge of the string ending on the O3-plane, has grading 0 .

From the quantization of each $Q_{a}^{(O)}$ we can fix the modulous in the orientifold to be

$$
\tau^{(O)}=\frac{1}{2}(1+i)
$$

Then $Q_{a}^{(O)}$ are given in the form $p+q \tau$, and $Q_{0}^{(O)}$, which has trivial grading, can be expressed as

$$
Q_{0}^{(O)}=p+2 q \tau^{(O)}, \quad p, q \in \mathbb{Z}
$$

This is nothing but the condition (2.8) for an $\widetilde{\mathrm{O}}^{-}{ }^{-}$-plane, which gives the gauge group SO(5).

Let us consider the other possibility: matching of two strings in (3.23) with $\boldsymbol{a}$ and $\boldsymbol{c}$ in (3.18). We obtain the relations

$$
z_{1}=-\frac{1}{2}(1-i) y_{1}+\frac{1}{2}(1+i) y_{2}, \quad z_{2}=\frac{1}{2}(1+i) y_{1}-\frac{1}{2}(1-i) y_{2},
$$

and

$$
Q_{1}^{(O)}=-\frac{1-i}{2} Q_{1}^{(S)}+\frac{1+i}{2} Q_{2}^{(S)}, \quad Q_{2}^{(O)}=\frac{1+i}{2} Q_{1}^{(S)}-\frac{1-i}{2} Q_{2}^{(S)},
$$

and the correspondents of $\boldsymbol{b}$ and $\boldsymbol{d}$ are the two remaining strings in (3.22). (See table 6.) 


\begin{tabular}{|ccccc|}
\hline & $Z^{(S)}$ & $\left(Q_{1}^{(S)}, Q_{2}^{(S)}\right)$ & $Z^{(O)}$ & $\left(Q_{1}^{(O)}, Q_{2}^{(O)}\right)$ \\
\hline $\boldsymbol{a}$ & $z_{1}-z_{2}$ & $(1,-1)$ & $-\left(y_{1}-y_{2}\right)$ & $(-1,1)$ \\
$\boldsymbol{b}$ & $(1-i)\left(z_{1}-i z_{2}\right)$ & $(1-i,-1-i)$ & $2 y_{2}$ & $(0,2)$ \\
$\boldsymbol{c}$ & $i\left(z_{1}+z_{2}\right)$ & $(i, i)$ & $-y_{1}-y_{2}$ & $(-1,-1)$ \\
$\boldsymbol{d}$ & $(1+i)\left(z_{1}+i z_{2}\right)$ & $(1+i,-1+i)$ & $-2 y_{1}$ & $(-2,0)$ \\
\hline
\end{tabular}

Table 6. The dyonic charges and the central charge of junctions in the S-fold and $\mathrm{O}^{+}{ }^{+}$and $\widetilde{\mathrm{O}} 3^{+}$-planes background are shown for four strings corresponding to W-bosons.

Substituting (3.26) into (3.31) we obtain

$$
Q_{1}^{(O)}=\frac{1+i}{2}\left[\left(p_{2}-q_{1}\right)+i\left(p_{1}+q_{2}\right)\right], \quad Q_{2}^{(O)}=\frac{1+i}{2}\left[\left(p_{1}-q_{2}\right)+i\left(p_{2}+q_{1}\right)\right] .
$$

These are similar to (3.27) and only difference up to unimportant signatures are the extra factors $1+i$. Therefore, the quantization and constraint for $Q_{a}^{(O)}$ can be obtained from the previous ones by simply rotate the lattice in figure 4 by 45 degrees and expand it by the factor $\sqrt{2}$. The resulting lattice is the same as figure 3. If we set $\tau^{(O)}=1+i$ the charges $Q_{a}^{(O)}$ take the form $p+q \tau^{(O)}$, and $Q_{0}^{(O)}$ can be expressed as

$$
Q_{0}^{(O)}=2 p+q \tau^{(O)}, \quad p, q \in \mathbb{Z} .
$$

This is the constraint $(2.8)$ for $\mathrm{O}^{+}$-plane. We can also set $\tau^{(O)}=i$. Then $Q_{0}^{(O)}$ can be expressed as

$$
Q_{0}^{(O)}=2 p+q\left(1+\tau^{(O)}\right), \quad p, q \in \mathbb{Z} .
$$

This is the constraint (2.8) for $\widetilde{\mathrm{O}}^{+}$-plane. These two assignments of $\tau^{(O)}$ give two $\operatorname{Sp}(2)$ theories with different $\theta$-angle, which are dual to each other.

In both cases, the spectrum of the central charge $Z$ in the S-fold matches completely with that of the orientifold.

Once we obtain these relations, we can rewrite $\bar{Z}$ as a function of the S-fold variables. For the $\mathrm{SO}(5)$ matching with (3.24) and (3.25) and the $\mathrm{Sp}(2)$ matching with (3.30) and (3.31) we obtain

$$
\mathrm{SO}(5): \bar{Z}=Q_{1}^{(S)} z_{2}^{*}+Q_{2}^{(S)} z_{1}^{*}, \quad \operatorname{Sp}(2): \bar{Z}=-Q_{1}^{(S)} z_{2}^{*}-Q_{2}^{(S)} z_{1}^{*}
$$

The overall sign depends on the choice of string orientation and is the matter of conventions. This result is the same as (3.12) up to sign.

As in the $k=3$ case let us illustrate the junctions corresponding to strings in S-fold. Strings connecting two independent D3-branes give W-bosons and they have been already analyzed above, and here we focus on strings connecting a D3-brane and one of its mirror branes. First we consider two strings

$$
z_{0, a} \stackrel{1}{\longleftarrow} z_{1, a}, \quad(a=1,2) .
$$




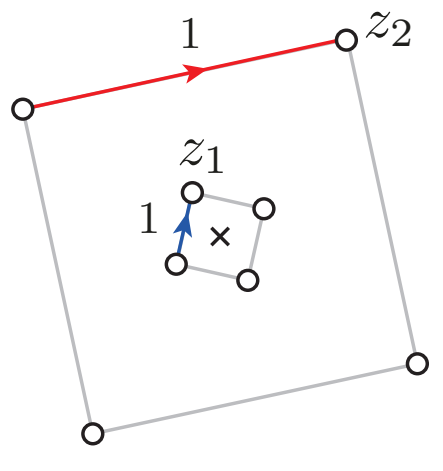

(a)

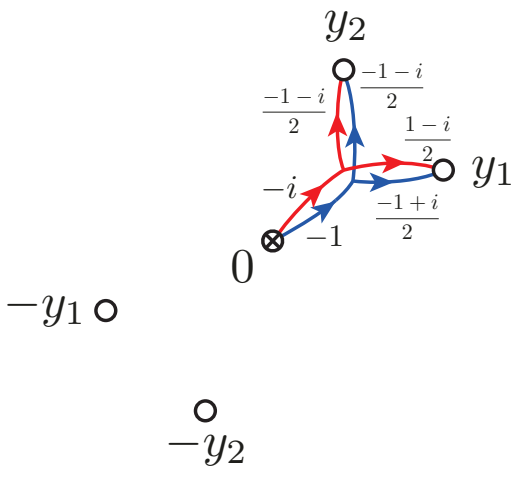

(b)

Figure 5. Examples of strings in the S-fold and corresponding junctions in the orientifold background.

These are shown in figure $5(\mathrm{a})$ in a situation with $\left|z_{1}\right| \ll\left|z_{2}\right|$. The dyonic charges of these strings are

$$
\left(Q_{1}^{(S)}, Q_{2}^{(S)}\right)=\left\{\begin{array}{ll}
(1-i, 0) & (a=1) \\
(0,1-i) & (a=2)
\end{array} .\right.
$$

By the relation (3.25) we obtain the charges of the corresponding junctions:

$$
\left(Q_{1}^{(O)}, Q_{2}^{(O)}\right)=\left\{\begin{array}{ll}
\left(\frac{-1+i}{2}, \frac{-1-i}{2}\right) & (a=1) \\
\left(\frac{1-i}{2}, \frac{-1-i}{2}\right) & (a=2)
\end{array} .\right.
$$

Junctions carrying these charges are shown in figure 5(b). As in figure 2 the red and blue junctions in figure 5(b) are holomorphic and anti-holomorphic, respectively.

Another pair of two strings in S-fold is

$$
z_{0, a} \stackrel{1}{\longleftarrow} z_{2, a}, \quad(a=1,2) .
$$

We can easily check that these strings carry dyonic charges that are as $1+i$ times as those in (3.37). The corresponding junctions are ones in figure 5(b) with charges multiplied by $1+i$.

\section{$3.3 \quad \mathbb{Z}_{6}$ S-fold}

For $k=6$ we expect $\mathcal{N}=4 \mathrm{SYM}$ with the gauge group $G_{2}$. The discrete torsion group for $k=6$ is trivial, and an arbitrary pair of the dyonic charges $\left(Q_{1}^{(S)}, Q_{2}^{(S)}\right) \in \Gamma_{6}+\Gamma_{6}$ is allowed. The central charge $Z$ is given by

$$
Z=Q_{1}^{(S)} z_{1}+Q_{2}^{(S)} z_{2}
$$

Although no perturbative realization of $G_{2}$ theory is known in string theory and we cannot directly read off the other central charge $\bar{Z}$ from junctions, it is natural to guess from the 
results for $k \leq 4$ that

$$
\bar{Z}=Q_{1}^{(S)} z_{2}^{*}+Q_{2}^{(S)} z_{1}^{*}
$$

up to overall phase. Indeed, if we assume

- $Z$ is given by $(3.40)$,

- $\bar{Z}$ is a holomorphic bilinear form of $Q_{a}^{(S)}$ and $z_{a}^{*}$. Namely, $\bar{Z}$ is given by $\bar{Z}=c_{a b} Q_{a}^{(S)} z_{b}^{*}$ with some coefficients $c_{a b}$, and

- $|Z|=|\bar{Z}|$ for charges satisfying the electric condition (3.3),

then (3.41) is the unique solution up to phase ambiguity.

\section{Walls of marginal stability}

In the previous sections we show that there are two types of $1 / 4$ BPS junctions: holomorphic and anti-holomorphic ones. They may decay or make the transition between two types of BPS states as we move D3-branes. In this section we demonstrate how this happens for some junctions. The purpose of this section is not to give a comprehensive analysis of general junctions but simply to show typical processes that occur for simple junctions when we move D3-branes. Thus, we consider only strings in S-folds that are shown in figure 2 and figure 5 .

Let us consider $\mathbb{Z}_{3}$ case first. As a starting point we take the red string and junction in figure 2 , which are also shown in figure 6 . As we previously mentioned the junction is holomorphic. Let $y_{*}$ be the intersection point of the junction. If $y_{*}$ coincide $0, y_{1}$, or $y_{2}$, the junction decays into a pair of strings. Let us first consider the wall with $y_{*}=0$. Because the angles at the junction point are all $2 \pi / 3, y_{*}=0$ is equivalent to

$$
y_{2}=t \omega y_{1}, \quad t \in \mathbb{R}_{+} .
$$

Using (3.10) we can rewrite this as the following relation between $z_{1}$ and $z_{2}$ :

$$
z_{1}=\frac{t}{t+1} \omega z_{2}+\frac{1}{t+1} \omega^{2} z_{2}
$$

which means $z_{1}$ is an internally dividing point of $\omega z_{2}$ and $\omega^{2} z_{2}$. If we illustrate relative position of D3-branes in the S-fold so that the position $z_{2}$ is fixed, this gives a wall of the transition on the $z_{1}$-plane. The other two walls $y_{*}=y_{1}$ and $y_{*}=y_{2}$ are given respectively by

$$
\begin{aligned}
-y_{1}=t \omega\left(y_{2}-y_{1}\right) & \leftrightarrow \quad z_{1}=\frac{t}{t+1} z_{2}+\frac{1}{t+1} \omega z_{2}, \\
y_{1}-y_{2}=t \omega\left(-y_{2}\right) & \leftrightarrow \quad z_{1}=\frac{t}{t+1} \omega^{2} z_{2}+\frac{1}{t+1} z_{2} .
\end{aligned}
$$

These three walls form the triangle with vertices at $z_{2}, \omega z_{2}$, and $\omega^{2} z_{2}$, which is shown as purple dashed lines in figure 6(a). The corresponding walls in the flat background are 
(a)

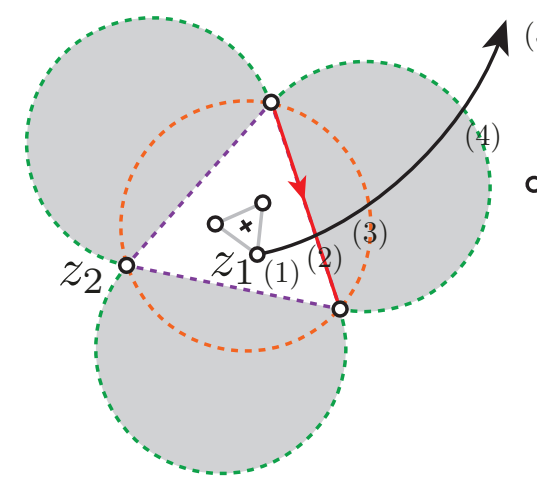

(b)

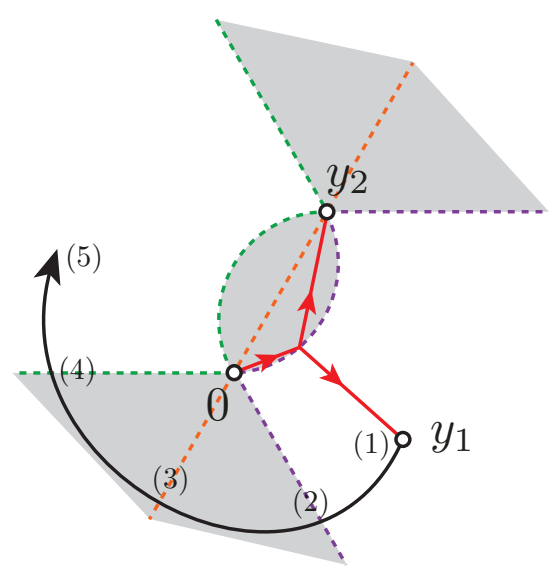

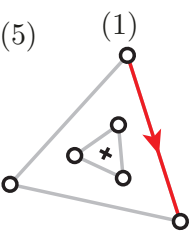

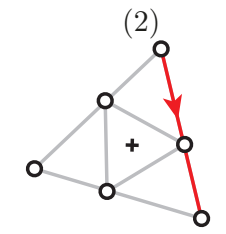

(3)

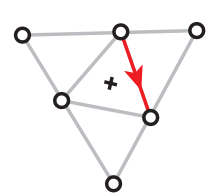

(4)

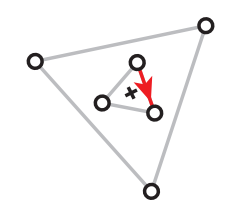

(5)

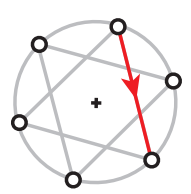

(1)

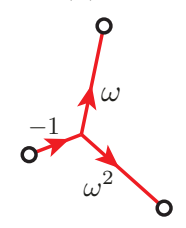

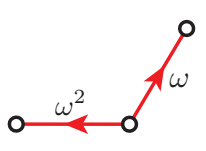

$(4)$
$(2)$

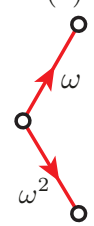

$(3)$

Figure 6. Moduli spaces for $\mathbb{Z}_{3}$ case are shown. $z_{2}, y_{2}$, and the origin are fixed and $z_{1}$ and $y_{1}$ moves. Walls, regions, and a typical example of wall crossing are illustrated.

shown in $6(\mathrm{~b})$ by the same color of dashed lines. When $y_{1}\left(z_{1}\right)$ crosses these walls the junction decays into two strings (see (b-2) in figure 6).

Let us continue the deformation. When $y_{2}=a y_{1}(a \in \mathbb{R})$ all D3-branes in the flat background are aligned on a line, and then the relation $\left|z_{1}\right|=\left|z_{2}\right|$ holds, which means D3branes in the S-fold are on a circle. (See orange dashed lines and (3) in figure 6.) Nothing special happens on this locus.

As $z_{1}$ goes further away from the origin, finally, it crosses the walls defined by

$$
\begin{array}{rlrl}
y_{1} & =t \omega y_{2} & \leftrightarrow & z_{1}=\omega^{2} z_{2} \frac{t+1}{t+\omega}, \\
y_{2}-y_{1}=t \omega\left(-y_{1}\right) & \leftrightarrow & z_{1}=\omega z_{2} \frac{t+1}{t+\omega}, \\
-y_{2}=t \omega\left(y_{1}-y_{2}\right) & \leftrightarrow & z_{1}=z_{2} \frac{t+1}{t+\omega},
\end{array}
$$

where $t$ is, again, real positive. These walls are shown as green dashed lines in figure 6 . Note that these equations also represent that $z_{2}$ is on a side of the triangle $z_{1}, \omega z_{1}$, and $\omega^{2} z_{1}$. After the crossing the pair of strings form an anti-holomorphic junction. (See (b-5) in figure 6.) 
(a)

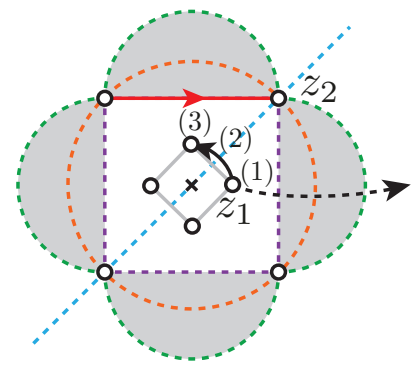

(1)

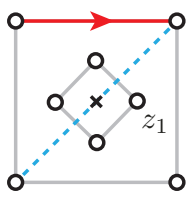

$(2)$

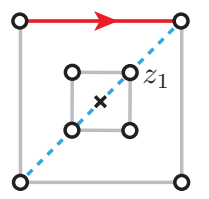

$(3)$

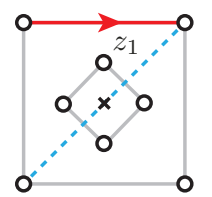

(b)

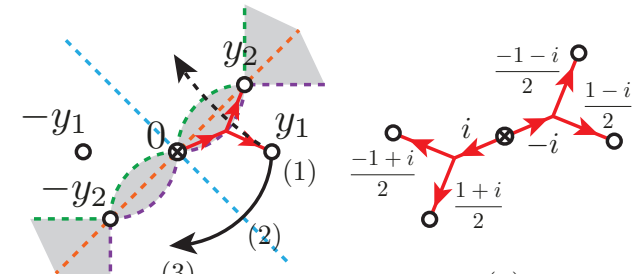

(3)

(1)

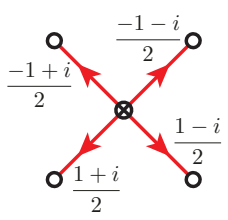

$(2)$

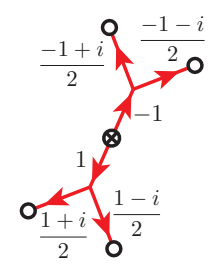

(3)

Figure 7. Moduli space for a string in $\mathbb{Z}_{4}$ S-fold is shown. $z_{2}, y_{2}$, and the origin are fixed and $z_{1}$ and $y_{1}$ moves. In the shaded regions the junction becomes non-BPS. The D3-brane motion shown by dashed arrows cause a deformation of the junction similar to what shown in figure 6 . Some string configurations in the motion along solid arrows are shown on the right.

Having illustrated the walls, it is clear that the gray regions in figure 6 are regions where the junctions cannot be sustained, hence, they are regions of non-BPS states. The other regions are ones of holomorphic or anti-holomorphic BPS states.

Let us move on to $\mathbb{Z}_{4}$ case as another example. We look into the red string and junction illustrated in figure 5. There are two different points compared to $\mathbb{Z}_{3}$ case. One is that the flat background in $\mathbb{Z}_{3}$ case is replaced by the orientifold. The other is that the angles at the junction point are not all $2 \pi / 3$ but $\pi / 2,3 \pi / 4$, and $3 \pi / 4$. By taking these into account we can easily determine the walls, and the results are illustrated in figure $7 .^{5}$

Let us follow a typical motion of D3-branes. We move the D3-brane at $z_{1}$ with $z_{2}$ kept intact. If we move $z_{1}$ outward as shown in figure 7 (a) by the dashed arrow, the deformation of the corresponding junction is quite similar to that of $\mathbb{Z}_{3}$ case, and we do not repeat the analysis for it. We comment on another type of wall that we do not meet in the $\mathbb{Z}_{3}$ case. It is illustrated as light-blue lines in figure 7. Let us move D3-branes following the solid arrows in the figure. In 7(b) we show both the junction and its mirror image by the reason which will become clear shortly. On the light-blue line each of the junction and its mirror becomes a pair of strings as in $7(\mathrm{~b}-2)$. If the brane pass through the light-blue line two strings ending on $y_{1}$ and $-y_{2}$ form a new junction. Although the topology of the junction changes in this process the junction keeps itself holomorphic.

\section{Discussions}

In this paper we investigated junctions in S-folds for the purpose of understanding the supersymmetry enhancement proposed by Aharony and Tachikawa. We determined the

\footnotetext{
${ }^{5}$ The analysis here is classical, and we simply assume the existence of states saturating the BPS bound for 3-pronged junctions in the orientifold.
} 
spectrum of dyonic charges of the theory from the information of junctions in the S-fold, and in the $k=3$ and $k=4$ cases we confirmed that the spectra are the same as those of $\mathcal{N}=4 \mathrm{SU}(3)$ and $\mathrm{SO}(5)$ gauge theories by using another perturbative brane realization of each theory. This agreement seems highly non-trivial, and gives a strong support to the realization of $\mathcal{N}=4$ supersymmetry.

There are many unsolved problems. What is the most desired would be a direct determination of the non-perturbative central charges $\bar{Z}$. Due to the lack of the information of $\bar{Z}$ we could not directly determine the masses of BPS states. When we established the correspondence of the spectrum of junctions and $\mathcal{N}=4$ dyonic particles, we use the information of one of the central charge $Z$, which can be seen perturbatively in the Sfold. However, to determine BPS saturating masses, we also need the other central charge $\bar{Z}$. Because this central charge is generated non-perturbatively together with the fourth supercharge in the supersymmetry enhancement, we cannot determine it by simply drawing the shapes of junctions.

There is another problem related to the marginal deformation. In this paper we showed that the spectrum of S-fold side gives dyonic spectrum of $\mathcal{N}=4$ theory with a particular value of the marginal deformation parameter $\tau$. However, if $\mathcal{N}=4$ is realized, we should be able to freely change the deformation parameter. The change of the parameter affects the central charges, and we should have the corresponding parameter on the S-fold side. The identification of the parameter in the S-fold is very important problem to understand the supersymmetry enhancement.

Even if we could obtain the central charges, it would be another problem to determine the BPS spectrum. The existence of BPS saturating states is highly non-trivial problem and we need to perform quantum analysis of the junctions to determine it. As far as we know this is open problem even for orientifolds.

In section 4 we studied wall crossing for simple string configurations. We determined walls by using another brane realization of the theory which is expected to be equivalent to the S-fold system. It is still unknown what happens on the S-fold side when the wall is crossed.

We believe any of these questions is important to understand dynamics of $\mathcal{N}=3$ theories and S-folds. We hope to return to these problems in future.

\section{Acknowledgments}

We would like to thank Tetsuji Kimura for valuable discussions. The work of YI is partially supported by Grand-in-Aid for Scientific Research (C) (No. 15K05044), Ministry of Education, Science and Culture, Japan. DY is supported by the ERC Starting Grant N. 304806, "The Gauge/Gravity Duality and Geometry in String Theory".

Open Access. This article is distributed under the terms of the Creative Commons Attribution License (CC-BY 4.0), which permits any use, distribution and reproduction in any medium, provided the original author(s) and source are credited. 


\section{References}

[1] O. Aharony and M. Evtikhiev, On four dimensional $N=3$ superconformal theories, JHEP 04 (2016) 040 [arXiv: 1512.03524] [INSPIRE].

[2] C. Cordova, T.T. Dumitrescu and K. Intriligator, Deformations of Superconformal Theories, arXiv: 1602.01217 [INSPIRE].

[3] I. García-Etxebarria and D. Regalado, $\mathcal{N}=3$ four dimensional field theories, JHEP 03 (2016) 083 [arXiv:1512.06434] [INSPIRE].

[4] S. Ferrara, M. Porrati and A. Zaffaroni, $N=6$ supergravity on Ad $S_{5}$ and the $\mathrm{SU}(2,2 / 3)$ superconformal correspondence, Lett. Math. Phys. 47 (1999) 255 [hep-th/9810063] [INSPIRE].

[5] J.H. Schwarz, Covariant Field Equations of Chiral $N=2, D=10$ Supergravity, Nucl. Phys. B 226 (1983) 269 [inSPIRE].

[6] J.H. Schwarz and P.C. West, Symmetries and Transformations of Chiral $N=2, D=10$ Supergravity, Phys. Lett. B 126 (1983) 301 [INSPIRE].

[7] O. Aharony and Y. Tachikawa, S-folds and $4 d \mathcal{N}=3$ superconformal field theories, JHEP 06 (2016) 044 [arXiv: 1602.08638] [INSPIRE].

[8] T. Nishinaka and Y. Tachikawa, On $4 d$ rank-one $\mathcal{N}=3$ superconformal field theories, JHEP 09 (2016) 116 [arXiv: 1602.01503] [INSPIRE].

[9] P.C. Argyres, M. Lotito, Y. Lü and M. Martone, Expanding the landscape of $\mathcal{N}=2$ rank 1 SCFTs, JHEP 05 (2016) 088 [arXiv: 1602.02764] [INSPIRE].

[10] J.H. Schwarz, Lectures on superstring and M-theory dualities: Given at ICTP Spring School and at TASI Summer School, Nucl. Phys. Proc. Suppl. 55B (1997) 1 [hep-th/9607201] [INSPIRE].

[11] K. Dasgupta and S. Mukhi, BPS nature of three string junctions, Phys. Lett. B 423 (1998) 261 [hep-th/9711094] [INSPIRE].

[12] A. Sen, String network, JHEP 03 (1998) 005 [hep-th/9711130] [INSPIRE].

[13] S.-J. Rey and J.-T. Yee, BPS dynamics of triple $(p, q)$ string junction, Nucl. Phys. B 526 (1998) 229 [hep-th/9711202] [INSPIRE].

[14] O. Bergman, Three pronged strings and $1 / 4$ BPS states in $N=4$ super Yang-Mills theory, Nucl. Phys. B 525 (1998) 104 [hep-th/9712211] [INSPIRE].

[15] E. Witten, Baryons and branes in anti-de Sitter space, JHEP 07 (1998) 006 [hep-th/9805112] [INSPIRE].

[16] Y. Imamura and S. Yokoyama, Superconformal index of $N=3$ orientifold theories, J. Phys. A 49 (2016) 435401 [arXiv: 1603.00851] [InSPIRE].

[17] Y. Hyakutake, Y. Imamura and S. Sugimoto, Orientifold planes, type-I Wilson lines and non-BPS D-branes, JHEP 08 (2000) 043 [hep-th/0007012] [INSPIRE].

[18] C. Fraser and T.J. Hollowood, Semiclassical quantization in $N=4$ supersymmetric Yang-Mills theory and duality, Phys. Lett. B 402 (1997) 106 [hep-th/9704011] [INSPIRE].

[19] K.-M. Lee and P. Yi, Dyons in $N=4$ supersymmetric theories and three pronged strings, Phys. Rev. D 58 (1998) 066005 [hep-th/9804174] [INSPIRE]. 
[20] A. Sen, BPS Spectrum, Indices and Wall Crossing in $N=4$ Supersymmetric Yang-Mills Theories, JHEP 06 (2012) 164 [arXiv:1203.4889] [INSPIRE].

[21] O. Bergman and B. Kol, String webs and 1/4 BPS monopoles, Nucl. Phys. B 536 (1998) 149 [hep-th/9804160] [INSPIRE].

[22] D. Bak, K. Hashimoto, B.-H. Lee, H. Min and N. Sasakura, Moduli space dimensions of multipronged strings, Phys. Rev. D 60 (1999) 046005 [hep-th/9901107] [INSPIRE]. 